\title{
Tortricidae (Lepidoptera) from Ethiopia, 2
}

\author{
J. Razowski, ${ }^{1}$ P. Trematerra² \\ 1 Institute of Systematics and Experimental Zoology, Polish Academy of Sciences, Kraków, Poland; \\ ${ }^{2}$ Department of Agriculture, Environment and Food Science, University of Molise, Campobasso, \\ Italy
}

\begin{abstract}
Twenty species of Tortricidae from Ethiopia, Oromia Region, are recorded of which Olethreutes didessae sp. n., Ancylis colaccii sp. n., and Gypsonoma giorgiae sp. n. are described as new; Eucosma thalameuta Meyrick, 1918, is transferred to the genus Cosmetra Diakonoff, 1977.
\end{abstract}

\section{Introduction}

The Tortricidae of Ethiopia are insufficiently known despite 80 years ago Meyrick (1932) published first faunistic paper on the Microlepidoptera of Ethiopia which included the data on several leafrollers. Then Razowski \& Trematerra (2010) recorded 36 species from this country of which 25 were newly described. They also characterised five collecting sites.

Based on so limited number of species there is no possibility to characterise the Ethiopian fauna. Razowski \& Trematerra (2010) mentioned only some species common to Ethiopia and the Republic of South Africa, Mozambique and Uganda. Razowski \& Wojtusiak (2012) found in Nigeria three species known from Ethiopia what is interesting zoogeographically. In the present collection there are further four species described or recorded from Nigeria (Lobesia hecista Razowski, L. talyana Razowski, L. lecta Razowski, and Endothenia gutturalis Meyrick, 1934) and found in Ethiopia. There are also some closely related species common to the two countries.

Correspondence: Pasquale Trematerra, Department of Agriculture, Environment and Food Science, University of Molise, Via de Sanctis, 86100 Campobasso, Italy. E-mail: trema@unimol.it

Key words: Ethiopia, faunistics, Lepidoptera, Tortricidae, new species.

Acknowledgements: the authors would like to thank Marco Colacci, Francesco Parisi, Andrea Sciarretta, (University of Molise, Italy) and Renato Regalin (University of Milano, Italy) for providing the material for the study.

Received for publication: 6 July 2012

Revision received: 7 August 2012.

Accepted for publication: 8 August 2012.

(C) Copyright J. Razowski and P. Trematerra, 2012

Licensee PAGEPress, Italy

Journal of Entomological and Acarological Research 2012; 44:e8

doi:10.4081/jear.2012.e8

This article is distributed under the terms of the Creative Commons Attribution Noncommercial License (by-nc 3.0) which permits any noncommercial use, distribution, and reproduction in any medium, provided the original author(s) and source are credited.

\section{Material and methods}

This paper is based on the material collected during two Italian expeditions in Oromia Region, southwestern Ethiopia, by the entomologists of the University of Molise and of the University of Milano. The expeditions were realized in November 2010 and January 2012. During 2010 the itinerary of the expedition was Wellega zone (Didessa River, $1280 \mathrm{~m}, 11-16.11 .2010$ ), and Ilubador zone (Dabeda River, $1800 \mathrm{~m}$, 14.11.2010). In 2012 the expedition visited Wellega zone (Didessa River, 1280 m, 24-28.01.2012), and Ilubador zone (Dabeda River, 1827 m, 26.01.2012; Bedelle, Mute Forest, 2060 m, 27.01.2012).

Tortricid adults were collected during the day by net and at night from a white sheet placed behind a 160 Watt mixed light. Genitalia were prepared using standard methods, the abdomen was macerated in $10 \%$ $\mathrm{KOH}$ and dissected under a stereoscopic microscope, the genitalia were separated and mounted in euparal on a glass slide. Adults and slides are deposited in P. Trematerra collection, Campobasso (Italy).

\section{Systematic part: Tortricidae}

\section{Subfamily Tortricinae}

Tribe Tortricini

Apotoforma fustigera Razowski, 1986 (Figures 1, 11)

MATERIAL EXAMINED. 1 male, Ethiopia, Wellega zone, Didessa River, 1280 m, 28.01.2012, Coord. UTM 37P 215814E, 961832N, legg. A. Sciarretta, F. Parisi.

REMARKS. A. fustigera was described from Mount Cameroon; the Ethiopian specimen has somewhat shorter subscaphium and the terminal process of the sacculus. Another closely related species, A. uncifera Razowski, 1964, is from Natal (Razowski, 1986).

Lozotaenia sciarrettae Razowski \& Termaterra, 2010

MATERIAL EXAMINED. 4 males and 3 females, Ethiopia, Ilubabor zone, Bedelle, Dabeta River, 1800 m, 14.11.2010, legg. A. Sciarretta, M. Colacci.

REMARKS. L. sciarrettae was described from the Bale Mountains, Ethiopia.

Metamesia episema Diakonoff, 1960

MATERIAL EXAMINED. 1 male, Ethiopia, Ilubabor zone, Bedelle, Dabeda River, 1800 m, 14.11.2010, legg. A. Sciarretta, M. Colacci. REMARKS. This species was described from Madagascar; recorded from Ethiopia by Razowski \& Trematerra (2010).

Choristoneura dinota (Meyrick, 1918) (Figures 2, 12) MATERIAL EXAMINED. 1 male, Ethiopia, Wellega zone, Didessa River, 1280 m, 16.11.2010, Coord. UTM 37P 215814E, 961832N, legg. A. Sciarretta, M. Colacci; 1 female, Ethiopia, Ilubabor zone, 
near Bedelle, Dabeda River, 1827 m, 26.01.2012, Coord. UTM 37P 201587E, 930253N, legg. A. Sciarretta, F. Parisi.

REMARKS. C. dinota was described from Malawi. It aso was recorded from Kilimanjaro, Tanzania (its synonymy Tortrix ptilocnemis Meyrick, 1920), Gabon, Kenya, and Congo.

Procrica ophiograpta (Meyrick, 1932)

MATERIAL EXAMINED. 1 male, Ethiopia, Ilubabor zone, Bedelle, Mute Forest, $2060 \mathrm{~m}, 27.01 .2012$, Coord. UTM 37P 207285E, 938068N, legg. A. Sciarretta, F. Parisi.
REMARKS. P. ophiograpta was described from Ethiopia and then recorded by Razowski \& Trematerra (2010) from Bale Mountaines (as P. ophiographa, sic!).

\section{Orilesa caminosa Razowski, 2012}

MATERIAL EXAMINED. 1 male, Ethiopia, Ilubabor zone, Bedelle, Dabeda River, 1800 m, 14.11.2010, legg. A. Sciarretta, M. Colacci; 1 male, Ethiopia, Wellega zone, Didessa River, 1280 m, 25.01.2012, Coord. UTM 37P 215814E, 961832N, legg. A. Sciarretta, F. Parisi. REMARKS. This species is described from Eala, Congo.
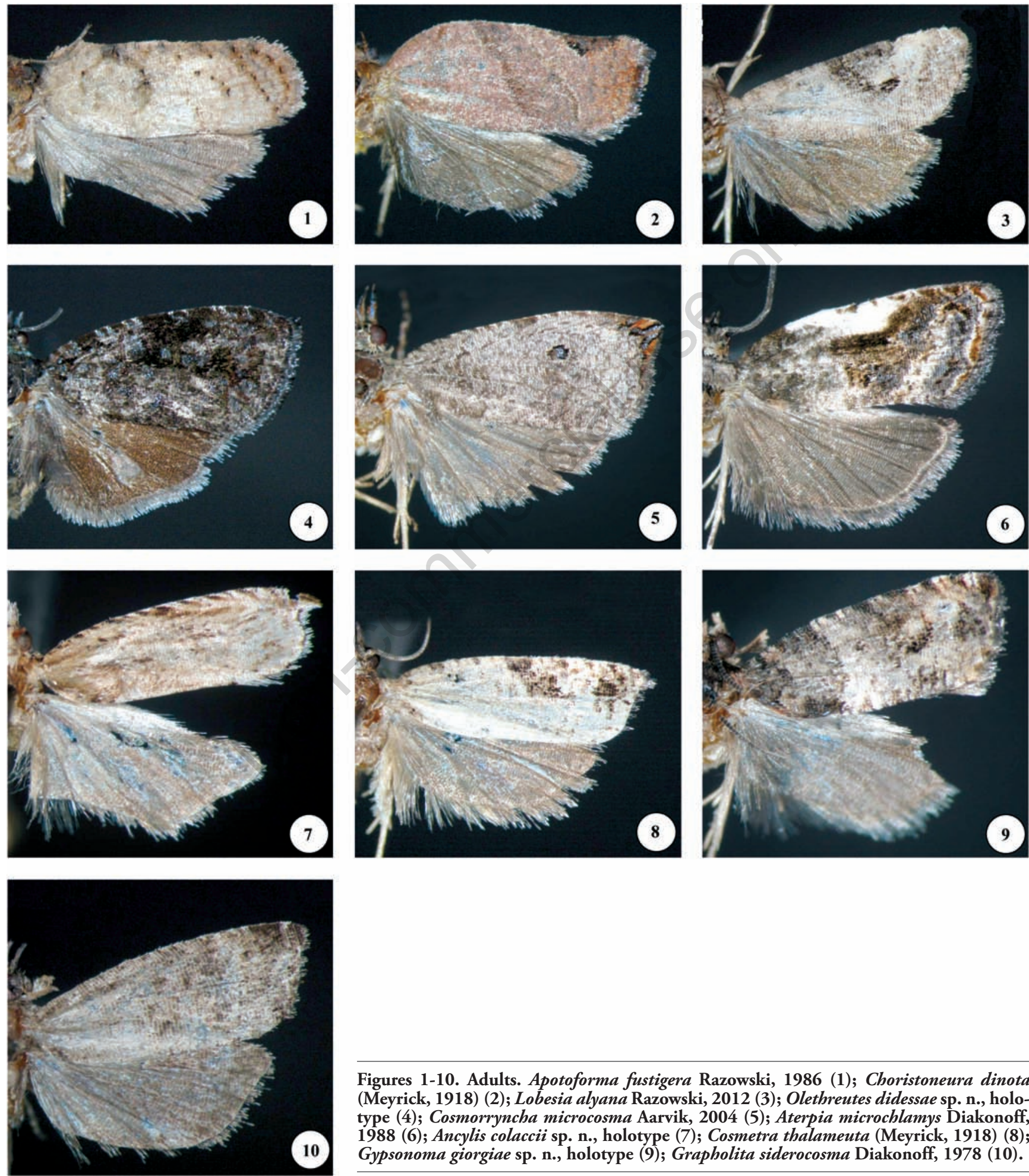

Figures 1-10. Adults. Apotoforma fustigera Razowski, 1986 (1); Choristoneura dinota (Meyrick, 1918) (2); Lobesia alyana Razowski, 2012 (3); Olethreutes didessae sp. n., holotype (4); Cosmorryncha microcosma Aarvik, 2004 (5); Aterpia microchlamys Diakonoff, 1988 (6); Ancylis colaccii sp. n., holotype (7); Cosmetra thalameuta (Meyrick, 1918) (8); Gypsonoma giorgiae sp. n., holotype (9); Grapholita siderocosma Diakonoff, 1978 (10). 
Tribe Bactrini

Endothenia ethiopica Razowski \& Trematerra, 2010

MATERIAL EXAMINED. 2 males, Ethiopia, Ilubabor zone, Bedelle, Mute Forest, $2060 \mathrm{~m}, 27.01 .2012$, Coord. UTM 37P 207285E, 938068N, legg. A. Sciarretta, F. Parisi; 1 male, Ethiopia, Ilubabor zone, near Bedelle, Dabeda river, 1827 m, 26.01.2012, Coord. UTM 37P 201587E, 930253N, legg. A. Sciarretta, F. Parisi.

REMARKS. This is an Ethiopian species described from Bale Mountains.

Endothenia albapex Razowski \& Trematerra, 2010 (Figure 13).

MATERIAL EXAMINED. 3 males, Ethiopia, Ilubabor zone, Bedelle, Mute Forest, $2060 \mathrm{~m}, 27.01 .2012$, Coord. UTM 37P 207285E, 938068N, legg. A. Sciarretta, F. Parisi; 1 female, Ethiopia, Ilubabor zone, near Bedelle, Dabeda River, 1827 m, 26.01.2012, Coord. UTM 37P 201587E, 930253N, legg. A. Sciarretta, F. Parisi.

DESCRIPTION. Female genitalia (Figure 13). Sterigma short, rounded; antrum sclerite broad, concave posteriorly; ductus bursae with subterminal cingulum; signum typical of the genus.

REMARKS. E. albapex was known to date only from Ethiopia (Bale Mountains). It was described in the genus Crotalaria Razowski \& Brown, [In Press]. The previous description (p. 56, figs 16, 59) is thus invalid; the above-mentioned characteristics are used in this new description in a valid genus.

\section{Tribe Olethreutini}

Lobesia hecista Razowski, 2012

MATERIAL EXAMINED. 1 male, Ethiopia, Wellega zone, Didessa River, 1280 m, 28.01.2012, Coord. UTM 37P 215814E, 961832N, legg. A. Sciarretta, F. Parisi.

REMARKS. This species was described from Nigeria.

Lobesia lecta Razowski, 2012

MATERIAL EXAMINED. 1 male, Ethiopia, Ilubabor zone, Bedelle, Mute Forest, $2060 \mathrm{~m}, 27.01 .2012$, Coord. UTM 37P 207285E, 938068N, legg. A. Sciarretta, F. Parisi.

REMARKS. In the male genitalia, L. lecta and L. hecista are similar to L. leucospilana (Mabille, 1900) from Madagascar but differ from it chiefly by a lack of a small lobe between end of the sacculus and ventral lobe of the cucullus.

Lobesia talyana Razowski, 2012 (Figures 3, 14)

MATERIAL EXAMINED. 1 male, Ethiopia, Wellega zone, Didessa River, 1280 m, 24.01.2012, Coord. UTM 37P 215814E, 961832N, legg. A. Sciarretta, F. Parisi.

REMARKS. L. talyana was described from Congo; besides it is known from Nigeria (Razowski, 2012).

Olethreutes didessae Razowski \& Trematerra, 2012 sp. n. (Figures 4, 15) MATERIAL EXAMINED. Holotype male, Ethiopia, Wellega zone, Didessa River, 1280 m, 26.01.2012, Coord. UTM 37P 215814E, 961832 N, legg. A. Sciarretta, F. Parisi.

DIAGNOSIS. $O$. didessae is closely related and similar to 0 . molybdachtha (Meyrick, 1930) from the Ivory Coast but didessae has broad uncus, large lateral socii, long slender terminal process of the sacculus and broad terminally lobe from the posterior edge of the basal cavity of the valva.

ETYMOLOGY. The specific epithet refers to the river of Didessa.

DESCRIPTION. Wing span $18 \mathrm{~mm}$. Head and thorax grey-black with black marks. Forewng fairly broad; costa uniformly convex; termen weakly obique and convex. Ground colour greyish with some whitish parts and bluish areas; suffusions blackish; costal strigulae small, white; divisions brown-black. Markings brownish black with black marks, diffuse, incomplete, consisting of basal blotch, median fascia, and subterminal fascia. Cilia grey-black. Hindwing brown with broadly rounded anal area; cilia whitish cream. Male genitalia (Figure 15). Uncus weakly sclerotized, helmet- shaped, long hairy; socius large, lateral, densely bristled; gnathos and tuba analis rather weakly sclerotized; sacculus with large, sharp termination, short caudal edge marked by broad, spiny lobe from base of which a slender process extends; neck of valva very slender; cuculus elongate; aedeagus short.

Female not known.

Cosmorryncha microcosma Aarvik, 2004 (Figures 5, 16)

MATERIAL EXAMINED. 1 male, Ethiopia, Wellega zone, Didessa River, 1280 m, 11.11.2010, Coord. UTM 37P 215814E, 961832N, legg. A. Sciarretta, M. Colacci.

REMARKS. C. microcosma was described from Congo (Zaire) (Aarvik, 2004), and is now known from Wellega zone, Ethiopia.

Aterpia microchlamys Diakonoff, 1983 (Figures 6, 17) MATERIAL EXAMINED. 1 male, Ethiopia, Ilubabor zone, Bedelle, Dabeda River, 1800 m, 14.11.2010, legg. A. Sciarretta, M. Colacci. REMARKS. A. microchlamys is described from Madagascar and is also recorded from Mt. Cameroon (Razowski, 2004).

\section{Tribe Enarmoniini}

Ancylis colaccii Razowski \& Trematerra, 2012 sp. n. (Figures 7, 18) MATERIAL EXAMINED. Holotype male, Ethiopia, Wellega zone, Didessa River, 1280 m, 11.11.2010, Coord. UTM 37P 215814E, 961832 N, legg. A. Sciarretta, M. Colacci.

DIAGNOSIS. In facies, A. colaccii is most similar to A. halisparta Meyrick, 1910, from Transvaal, South Africa but colaccii has whitish vertex of the head (in halisparta it is rust); from A. oculifera (Walsingham, 1891) from Bathurst, Gambia, colaccii differs by its strongly sinuate termen of forewing and anal edge of the hindwing, and a lack of brown oval spot at base of the apex (both compared species are known only from females). The male genitalia of this species differ from all known congeners by a ventral convexity of the base of the cucullus.

ETYMOLOGY. The species is named after his collector dr Marco Colacci (University of Molise, Campobasso, Italy).

DESCRIPTION. Wing span $14 \mathrm{~mm}$. Head whitish cream, labial palpus whitish cream sprinkled brown; thorax cream, tegulae brownish. Forewing slender; costa weakly convex; apex elongate-pointed; termen sinuate. Ground colour cream sprinkled and suffused brown and brown ferruginous; costal strigulae whitish, divisions rust brown and brownish. Remnants of markings brownish rust. Cilia cream. Hindwing brownish; cilia (rubbed) cream.

Male genitalia (Figure 18). Uncus reduced; socius broad, densely hairy; basal part of valva broad; neck slender expanding posterad; sacculus convexly rounded; cucullus convex ventrobasally, with slight submedian convexity; aedeagus long, slender, indistinctly tapering terminad, cornuti long and slender.

Female not known.

\section{Tribe Eucosmini}

Cosmetra thalameuta (Meyrick, 1918), comb. n. (Figures 8, 19) MATERIAL EXAMINED. 1 male, Ethiopia, Ilubabor zone, Bedelle, Mute Forest, $2060 \mathrm{~m}, 27.01 .2012$, Coord.UTM 37P 207285E, 938068N, legg. A. Sciarretta, F. Parisi.

REMARKS. C. thalameuta was described in the genus Eucosma Hübner, [1825] from Pretoria, South Africa. The type was illustrated by Razowski \& Krüger (2007).

Gypsonoma giorgiae Razowski \& Trematerra, 2012 sp. n. (Figures 9, 20) MATERIAL EXAMINED. Holotype male, Ethiopia, Ilubabor zone, Bedelle, Dabeda River, 1800 m, 14.11.2010, legg. A. Sciarretta, M. Colacci. 1 male, Ethiopia, Ilubabor zone, Bedelle, Dabeda River, $1800 \mathrm{~m}, 14.11 .2010$, legg. A. Sciarretta, M. Colacci.

DIAGNOSIS. In facies, $G$. giorgiae is similar to $G$. opsonoma (Meyrick, 1918) from Transvaal, the Republic of South Africa but giorgiae has brown forewing markings; male genitalia of giorgiae 


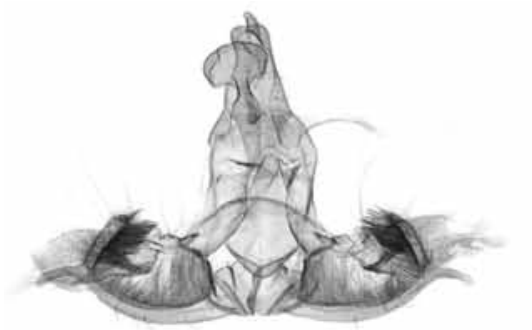

11

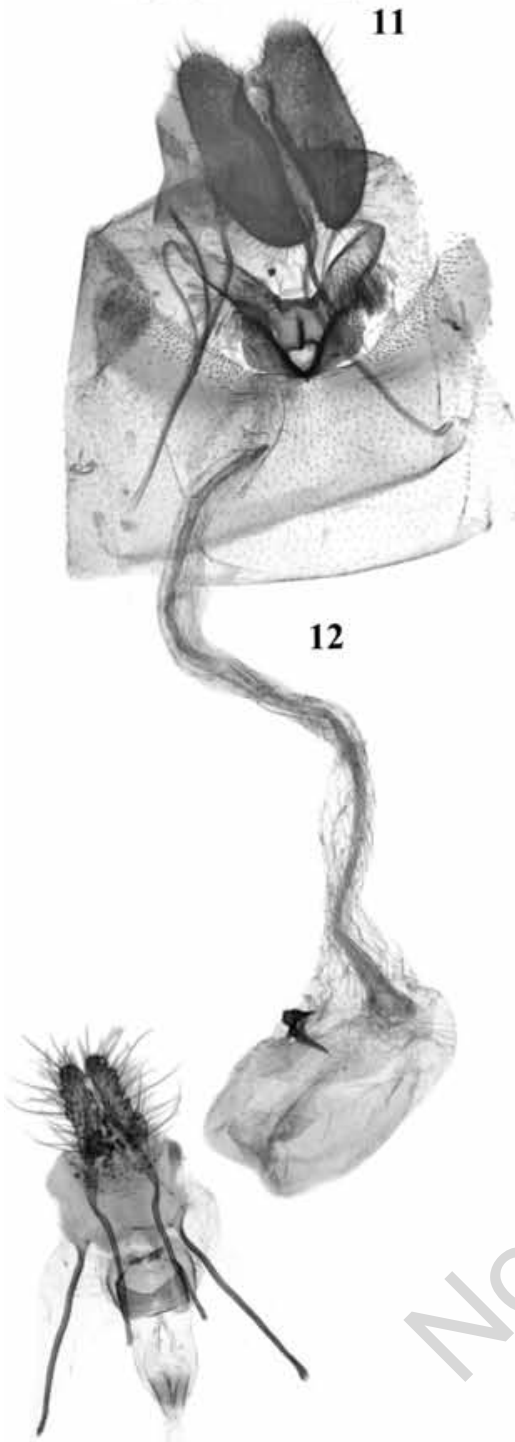

13

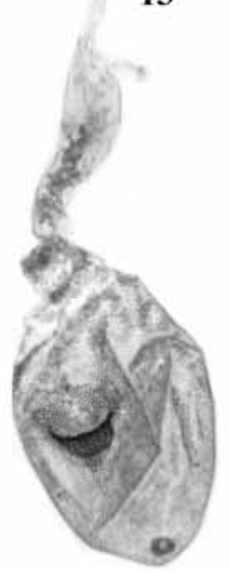

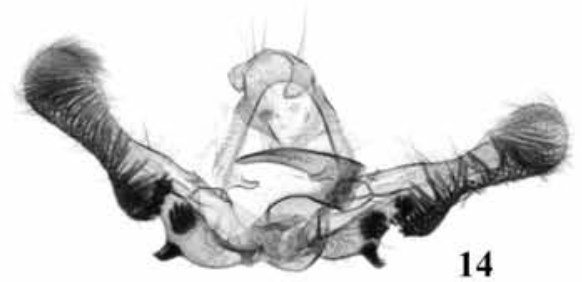

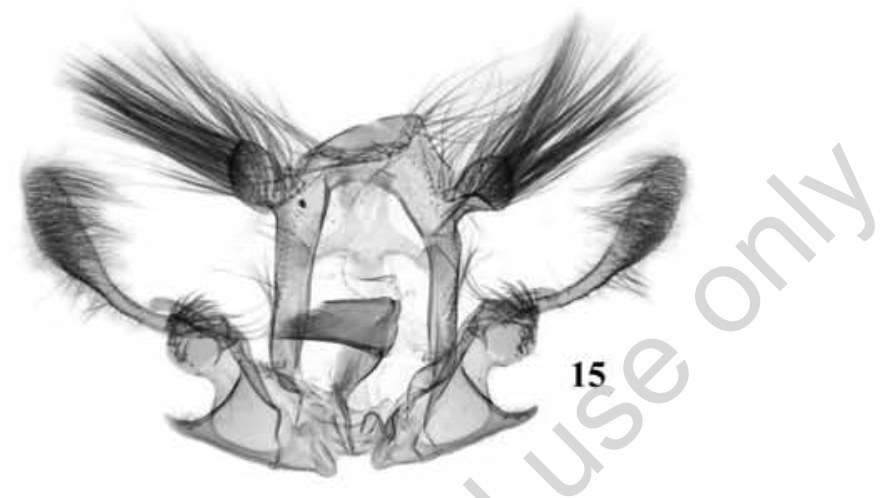

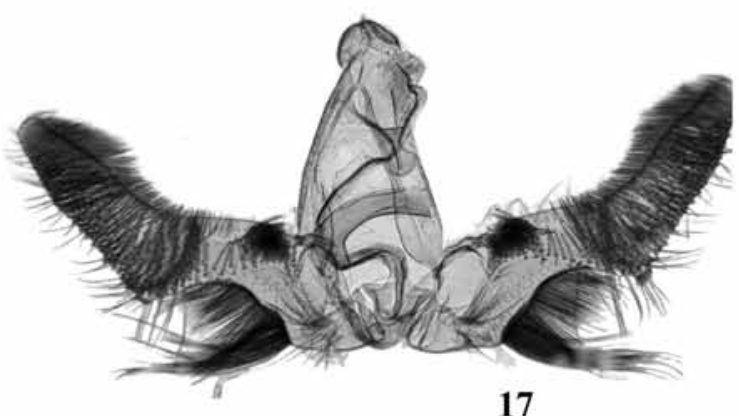

17
Figures 11-17. Genitalia. Apotoforma fustigera Razowski, 1986 (11); Choristoneura dinota (Meyrick, 1918) (12); Endothenia albapex Razowski \& Trematerra, 2010 (13); Lobesia alyana Razowski, 2012 (14); Olethreutes didessae sp. n., holotype (15); Cosmorryncha microcosma Aarvik, 2004 (16); Aterpia microchlamys Diakonoff, 1988 (17). 

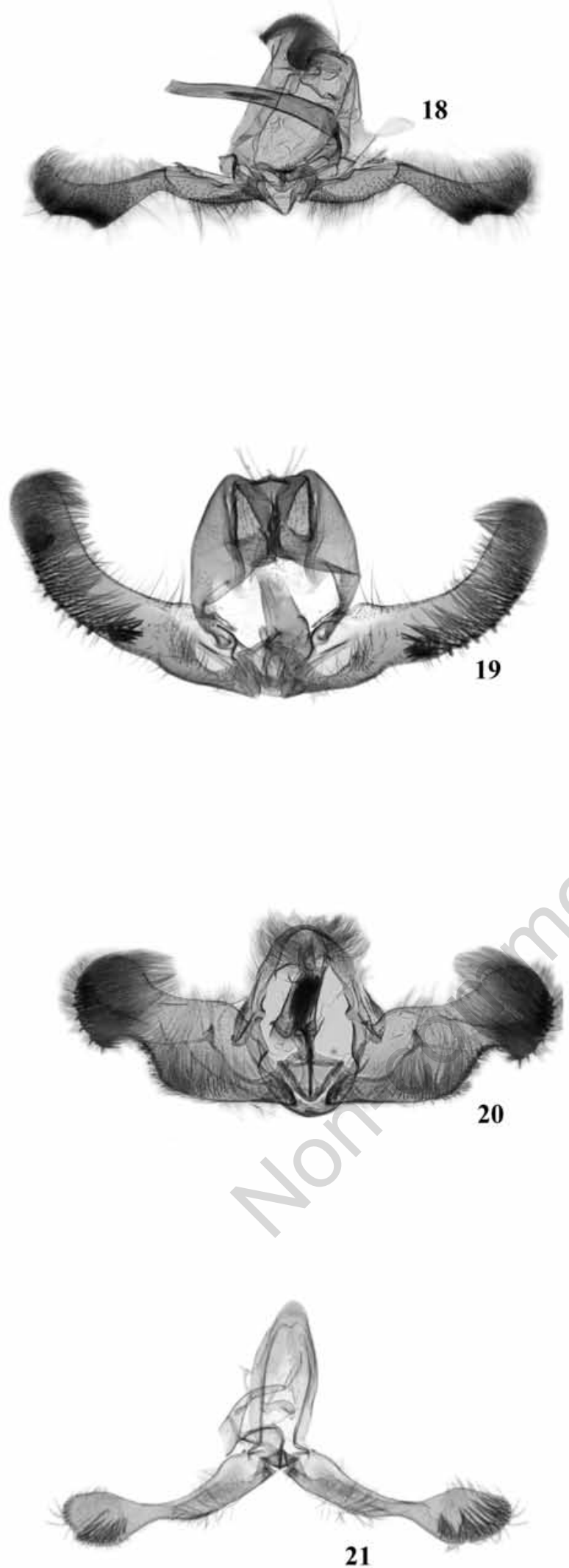

Figures 18-21. Genitalia. Ancylis colaccii sp. n., holotype (18); Cosmetra thalameuta (Meyrick, 1918) (19); Gypsonoma giorgiae sp. n., holotype (20); Grapholita siderocosma Diakonoff, 1978 (21). differ from other species in slender horn on distal edge of the basal cavity, distinct ventral incision of the valva, and a reduced membranous outer part of the cucullus (Figure 20).

ETYMOLOGY. This species is named after the daughter of the younger author.

DESCRIPTION. Wing spam 13-14 mm. Head grey-brown, labial palpus brownish; thorax brown with black-brown marks. Forewing hardly expanding terminad; costa slightly convex; termen indistinctly concave medially, rather not oblique. Ground colour white, tinged yellowish terminally; strigulation and suffusions brown; costal strigulae white, divisions blackish brown; apex concolorous with the latter marked rust posteriorly. Markings blackish brown with blackish parts; basal blotch fused with postbasal fascia; median fascia atrophying subdorsally; subterminal blotch atrophied towards costa. Cilia brownish. Hindwing brownish; cilia cream.

Male genitalia (Figure 20). Uncus tapering terminad; socius broad, curved; valva fairly slender; sacculus simple; neck rudimentary; cucullus uniformly broad with ventroproximal group of spines; aedeagus short.

Tribe Grapholitini

Cydia tytthaspis Razowski \& Trematerra, 2010

MATERIAL EXAMINED. 1 male, Ethiopia, Ilubabor zone, near Bedelle, Dabeda River, 1827 m, 26.01.2012, Coord. UTM 37P 201587E, 930253N, legg. A. Sciarretta, F. Parisi.

REMARKS. C. tytthaspis was described from Bale Mountains where it was collected at $3100 \mathrm{~m}$.

Thaumatotibia batrachopa (Meyrick, 1908)

MATERIAL EXAMINED. 1 male, Ethiopia, Ilubabor zone, Bedelle, Dabeda River, 1800 m, 14.11.2010, legg. A. Sciarretta, M. Colacci. REMARKS. T. batrachopa was described from Cape Colony, South Africa. It also was recorded from Bale Mountains, Ethiopia (Razowski \& Trematerra 2010).

Grapholita siderocosma Diakonoff, 1978 (Figures 10, 21)

MATERIAL EXAMINED. 1 male, Ethiopia, Wellega zone, Didessa River, 1280 m, 24.01.2012, Coord. UTM 37P 215814E, 961832N, legg. A. Sciarretta, F. Parisi.

REMARKS. G. siderocosma was described from the Réunion Island.

\section{References}

AARVIK L., 2004 - Revision of the subtribe Neopotamiae (Lepidoptera: Tortricidae) in Africa. - Norw. J. Entomol. 51: 71-122.

MEYRICK E., 1932 - Entomological expedition to Abyssinia, 1926-7. Microlepidoptrera. - Trans. Ent. Soc. London 80: 107-120.

RAZOWSKI J., 1986 - The data on Tortricini (Lepidoptera, Tortricidae) published after 1966. - Acta Zool. Cracov. 29: 423-440.

RAZOWSKI J., 2004 - Review of the genera of Afrotropical Tortricidae (Lepidoptera). - Acta Zool. Cracov. 47: 167-210.

RAZOWSKI J., 2012 - Tortricidae from the Tervuren Museum, 2: Olethreutini (Insecta: Lepidoptera). - Genus 2: 163-182.

RAZOWSKI J., KRÜGER M., 2007 - An illustrated catalogue of the type specimens of Tortricidae in the Transvaal Museum, Pretoria (Lepidoptera: Tortricidae). - SHILAP Revta Lepid. 35: 103-179.

RAZOWSKI J., TREMATERRA P., 2010. - Tortricidae (Lepidoptera) from Ethiopia. - J. Entomol. Acarol. Res. Ser. II 42: 47-79.

RAZOWSKI J., WOJTUSIAK J., 2012. - Tortricidae (Lepidoptera) from Nigeria. - Acta Zool. Cracov. [In Press]. 